Return to the Manage Active Submissions page at http://spie.org/submissions/tasks.aspx and approve or disapprove this submission. Your manuscript will not be published without this approval. Please contact author_help@spie.org with any questions or concerns.

\title{
Expanded beam spectro-ellipsometry for big area on-line monitoring
}

\author{
M. Fried $*^{\mathrm{a}}$, C. Major ${ }^{\mathrm{a}}$, G. Juhasz ${ }^{\mathrm{a}}$, P. Petrik ${ }^{\mathrm{a}}$, Z. Horvath
}

anstitute for Technical Physics and Materials Science (MTA EK MFA), H-1525 Budapest, POB 49, Hungary; ${ }^{b}$ Institute for Solid State Physics and Optics, Wigner Research Centre for Physics (MTA Wigner SZFI), H-1525 Budapest, POB 49, Hungary

\begin{abstract}
Non-destructive analysing tools are needed at all stages of thin film process-development, especially photovoltaic (PV) development, and on production lines. In the case of thin films, layer thicknesses, micro-structure, composition, layer optical properties, and their uniformity are important parameters. An important focus is to express the dielectric functions of each component material in terms of a handful of wavelength independent parameters whose variation can cover all process variants of that material. With the resulting database, spectroscopic ellipsometry coupled with multilayer analysis can be developed for on-line point-by-point mapping and on-line line-by-line imaging. Off-line point-by-point mapping can be effective for characterization of non-uniformities in full scale PV panels or big area (even $450 \mathrm{~mm}$ diameter) Si-wafers in developing labs but it is slow in the on-line mode when only 15 points can be obtained (within $1 \mathrm{~min}$ ) as a $120 \mathrm{~cm}$ long panel moves by the mapping station. Last years [M. Fried et al, Thin Solid Films 519, 2730 (2011)], a new instrumentation was developed that provides a line image of spectroscopic ellipsometry (wl=350$1000 \mathrm{~nm}$ ) data. Earlier a single 30 point line image could be collected in $10 \mathrm{~s}$ over a $15 \mathrm{~cm}$ width of PV material. Recent years we have built a 30, a 45 and a $60 \mathrm{~cm}$ width expanded beam ellipsometer which speed is increased by 10x. Now, 1800 points can be mapped in a 1 min traverse of a $60 * 120 \mathrm{~cm} \mathrm{PV}$ panel or flexible roll-to-roll substrate.
\end{abstract}

Keywords: spectro-ellipsometry, mapping, on-line monitoring

e-mail: fried@mfa.kfki.hu

\section{INTRODUCTION}

Production and installation of PhotoVoltaic (PV) modules grew exponentially in the last decade. The thin film PV production and installation has grown similarly, or even faster, because its market share has grown from $5 \%$ upto $15 \%$. Presently, CdTe and CuInGaSe2 (CIGS) based thin film PV-s are overgrown the a-Si type PV-s. The best and average efficiences of all types of PV-modules are growing continuosly. At the same time, the relative fabrication cost (i.e. price of Wattpeak) and energy pay-back time continuosly reducing and one can see from the so called "price learning curves" that the different thin film technologies are well ahead in cost effectiveness against the bulk crystalline Si-technologies. [Photovoltaics Report (Roland Schindler (Fraunhofer ISE) and Werner Warmuth (PSE AG)), Freiburg, October 24, 2014, www.ise.fraunhofer.de, FRAUNHOFER INSTITUTE FOR SOLAR ENERGY SYSTEMS ISE]

Longitudinal elementary cells are connected electrically in series in thin film solar panels and the module power is limited by the elementary cell with the lowest current, so structural uniformity is essential to achieve high efficiency. If we want to close the gap between „Lab cell” and „Industrial module” efficiency, the key factor to increase the efficiency in the case of thin-film solar panels or modules is the lateral homogeneity.

\section{SPECTROSCOPIC ELLIPSOMETRY (SE) IN PHOTOVOLTAICS}

The last decades have seen widespread use of SE in different fields of research. Ellipsometry offers great promise for characterization, monitoring, and control of a wide variety of processes, especially in semiconductor related areas [1-12]

\subsection{Micromorph silicon solar cell structures}

One can spatially correlate the properties of the hydrogenated silicon (a-Si:H) i- and p-layers-as mapped over a larger substrate area-with device performance parameters from an array of a-Si:H based n-i-p dot cells. Analysis of the SE data over the full area provides maps of i-layer thickness and band gap, p-layer thickness and band gap, and p-layer surface roughness thickness for the n-i-p solar cell structure. The mapped values adjacent to the devices can be correlated with photovoltaic (PV) device performance parameters. When sufficient nonuniformity exists, these correlations enable optimization based on specific values of the fundamental properties. Alternatively, if the optimum set of properties has been identified, the impact of deviations due to macroscopic uniformities can be evaluated. [13] 
Return to the Manage Active Submissions page at http://spie.org/submissions/tasks.aspx and approve or disapprove this submission. Your manuscript will not be published without this approval. Please contact author_help@spie.org with any questions or concerns.

\subsection{CIGS solar cell structures}

In the case of CIGS $\left(\mathrm{Cu}\left(\mathrm{In}_{1-\mathrm{x}} \mathrm{Ga}_{\mathrm{x}}\right) \mathrm{Se}_{2}\right)$ the compositional change is an additional parameter. SE has the potential to map the alloy composition, the bulk layer and the surface roughness layer thicknesses of CIGS thin films. As a result, the methodology is suitable for characterization in online production-level applications. In order to develop the mapping capability, CIGS films having different molar Ga contents $\mathrm{x}$ and fixed copper stoichiometry were deposited and measured in situ by SE in order to extract the complex dielectric functions $\left(\varepsilon=\varepsilon_{1}+\mathrm{i} \varepsilon_{2}\right)$ of these films. [14] The $\left(\varepsilon_{1}, \varepsilon_{2}\right)$ spectra were parameterized using an oscillator sum. Best-fitting equations were obtained that relate each oscillator parameter to the Ga content $\mathrm{x}$, as determined by energy dispersive X-ray analysis. This approach reduces the number of fitting parameters for $\left(\varepsilon_{1}, \varepsilon_{2}\right)$ from several to just one: the Ga content x. [15-18] Because $\left(\varepsilon_{1}\right.$, $\left.\varepsilon_{2}\right)$ is now represented by this single parameter, the chances of parameter correlations during fitting are reduced, enabling production-scale compositional mapping of chalcopyrite films by SE.

\subsection{Broadening of optical transitions in polycrystalline CdS and CdTe thin films}

The dielectric functions of polycrystalline CdS and CdTe thin films can be measured by in situ SE. Differences in due to processing variations are well understood using an excited carrier scattering model. A carrier mean free path can be defined that is found to be inversely proportional to the broadening of each of the band structure critical points (CPs). With this database, dielectric functions can be analyzed to evaluate the quality of materials used in CdS/CdTe photovoltaic heterojunctions. [19, 20]

\subsection{Transparent Conductive Oxides (TCO)}

Using appropriate optical models, the characterization of the layer thickness and carrier properties becomes possible from SE analysis, even for textured surfaces.

\section{SE analysis of textured $\mathrm{SnO}_{2}: F$ substrate}

The dielectric functions of the bulk $\mathrm{SnO}_{2}: \mathrm{F}$ layers are well represented using a Tauc-Lorentz oscillator term to describe the interband transitions and a Drude term to describe the free electrons. [21, 22] In order to express the complicated optical response in the textured structures, the optical model can incorporate (i) the surface roughness and interface layers calculated using an effective-medium-approximation multilayer model and (ii) the a-Si: $\mathrm{H} / \mathrm{SnO} \mathrm{O}_{2}: \mathrm{F}$ structure divided into two regions with different thicknesses. [23]

\section{SE analysis of ZnO:Al}

$\mathrm{SE}$ measurements can be used to measure the dielectric function of the $\mathrm{ZnO}$ :Al films. Monitoring of the electrical properties of Al-doped $\mathrm{ZnO}$ layers by SE measurements are feasible by using the analytical expression suggested by Yoshikawa and Adachi [24], and the results show correlation between specific resistance and band gap energy and direct exciton strength parameter. [25]

\subsection{Characterization of $\mathrm{SiH}_{2}$ content in a-Si:H}

For hydrogenated amorphous silicon (a-Si:H) layers prepared by plasma-enhanced chemical vapor deposition, $\mathrm{SiH}_{2}$ bonds exist at the internal surface of the void-rich structure and light absorption reduces due to the void formation. The amplitude of the $\varepsilon_{2}$ spectra obtained from various a-Si:H layers is expressed completely by the $\mathrm{SiH}_{2}$ bond density in the a-Si:H and reduces strongly with increasing the $\mathrm{SiH}_{2}$ content, indicating that microvoids present in the a-Si:H network are surrounded by the $\mathrm{SiH}_{2}$ bonding state. Validity of $\mathrm{SiH}_{2}$ analysis can be confirmed from IR ellipsometry. [26]

\section{FAST SE-MAPPING BY EXPANDED BEAM ELLIPSOMETRY}

In photovoltaic (PV) production for commercialization many key problems are related to scale-up. The difference is primarily caused by fluctuations in area uniformity of properties caused by the individual processing steps. The primary problem with many mapping probes [e.g., single-spot spectroscopic ellipsometry (SE), Kelvin probe, laser beam induced currents, etc.] is their long measurement time that makes in-line mapping impossible. We developed and (in this work) we demonstrate a high-speed and high-resolution measurement method to monitor the thin film processes in PV or ICtechnology in-line over large areas.

Ellipsometry determines angle-of-incidence dependent relative amplitude ratios and phase difference shifts upon specular reflection of light from a planar surface. Thus, collimated light beams are conventionally used with a well 
Return to the Manage Active Submissions page at http://spie.org/submissions/tasks.aspx and approve or disapprove this submission. Your manuscript will not be published without this approval. Please contact author_help@spie.org with any questions or concerns.

defined angle of incidence at the reflecting surface. Here we present an ellipsometric method fundamentally different from the conventional techniques [27-32]. In our instrument, the sample is illuminated by an almost diffuse, "divergent beam" of light, providing a collection of rays with diverse angles of incidence at every point of the sample. Precise "angle-selection" is performed on the detector side by a pin-hole camera. The pin-hole works as an "angle-of-incidence filter", selecting only one single light-beam from every direction (or sample position). The angular resolution of this type of camera is dependent on the diameter of the pin-hole. In the case of a single wavelength measurement, this instrument solution is appropriate for measuring a large area sample with possible non-uniform properties such that the pinhole camera selects a single angle of incidence corresponding to each sample point, but that angle varies across the sample. If the sample is moved parallel to its surface along a line during the measurement, however, each sample point will experience multiple angles of incidence successively, which provides additional information to assist in data analysis. By repeating the measurement with light of various wavelengths, multi-angle, multi-wavelength (but not continuous spectroscopic) ellipsometry data can be acquired; see ref. [28, 29].

By confining the number of measured sample points to a narrow range along a line only, we can generate continuous spectroscopic data at each point along the line image using white light illumination with a spectral dispersion (grating) after the pin-hole. This instrument produces spatial information in one dimension of the CCD array simultaneously with spectroscopic information in the perpendicular direction of the array; see Fig. 1. Both ellipsometric approaches (multiangle/multi-wavelength and continuous spectroscopic) are useful, for example, in the analysis of product moving along a coating line.

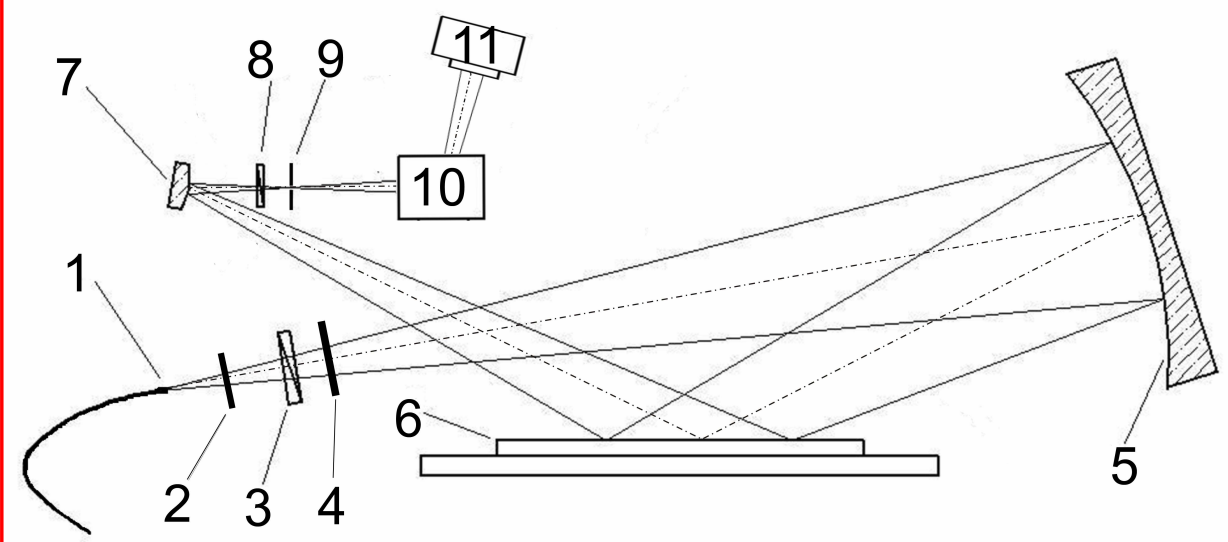

Figure 1: (1) "white" light source, (2) narrow, rectangular aperture, (3) film-polarizer, (4) compensator, (5) spherical mirror (6) sample, (7) cylindrical mirror, corrected beam, (8) analyzer, (9) pinhole, (10) correction-dispersion optics, (11) CCD detector array.

\section{INSTRUMENTATION}

A near-ultraviolet-to-visible (NUV-Vis) range $(350-630 \mathrm{~nm})$ of the first generation divergent beam instrument [31, 32] were built in MFA, Budapest, but this prototype limits potential photovoltaics applications; as a result, an extension into the near-infrared (NIR) region were performed to probe below the band gap of absorber layers in order to measure their thicknesses [32]. Thus, with a broadened spectral range, it became possible to characterize a wider variety of layers and structures. Divergent beam ellipsometers (using uncollimated beam) employ film polarizers, which exhibit a wide semifield angle but limited spectral range. In earlier state, dual spectral range capability was a convenient solution whereby the optical elements (source, polarizer-analyzer pairs, and optical grating) were automatically interchangeable, and the entire nuv-nir (350-1000 nm) spectra for a line image was detectable in two measurement cycles with one CCD camera [32].

Calibrations of the angle of incidence (relationship of pixel number to angle) and the mirror effects are performed via well-known and optimized structures, in this case three $\mathrm{SiO}_{2} / \mathrm{Si}$ samples with different thicknesses. Although the precision and accuracy of the instrument is not higher than that of standard step-wise wavelength scanning ellipsometers that probe a single spot, it is capable of determining the thickness of a silicon-dioxide film with sub-nanometer precision 
Return to the Manage Active Submissions page at http://spie.org/submissions/tasks.aspx and approve or disapprove this submission. Your manuscript will not be published without this approval. Please contact author_help@spie.org with any questions or concerns.

and the angle-of-incidence with sub-tenth-degree precision in the calibration process. Mirror and possible window effects are calculated using the following equation:

$$
\rho_{\mathrm{opt}}=\rho_{\text {meas }} * \rho_{\text {mirror }} \text { (different for each point and each wavelength) }
$$

where $\rho_{\text {opt }}$ is the measured value without mirrors and windows, $\rho_{\text {meas }}$ is the actually measured value, and $\rho_{\text {mirror }}$ is the effect of the mirrors and the windows of the chamber (if any). The calibration can be performed independently for each pixel of the CCD; however, improved signal-to-noise is obtained by summing the irradiances according to pixel-groups, in consideration of the lateral resolution of the system. A smooth variation is expected for the angle of incidence and for the $\left\{\operatorname{Re}\left(\rho_{\text {mirror }}\right), \operatorname{Im}\left(\rho_{\text {mirror }}\right)\right\}$ values. As a result, smoothing of the dependence of angle of incidence on position as well as the dependence of $\rho_{\text {mirror }}$ on position and wavelength reduces errors in the calibration measurement [32].

Rotating compensator measuring mode can enhance the performance of multi channel mapping ellipsometry, comparing with rotating polarizer or analyzer measuring mode. After careful calibration (most part of the beam is going through at non-normal direction through the compensator) we have the angle of incidences, the mirror-(window)-corrections and the $\delta$-shift by the retarder at all sample(-CCD)-points. [32]

\section{DEMONSTRATION SAMPLE PREPARATIONS}

Thermal oxide layers on 6-inch Si-wafers were prepared in usual furnace for calibrating and validating measurements. Poly-Si layer on $\mathrm{SiO}_{2}$ on 6-inch Si-wafer (poly-Si 46) were prepared by LPCVD.

$\mathrm{ZnO}$ layer were deposited by magnetron sputtering onto Ag-covered plastic foil in a cassette roll-to-roll model machine.

Hydrogenated amorphous silicon (a-Si:H), nanocrystalline silicon (nc-Si:H), and mixed phase layers were deposited by very high frequency plasma enhanced chemical vapor deposition (vhf PECVD) onto $\sim 15 \mathrm{x} 15 \mathrm{~cm}^{2}$ soda lime glass (SLG) and $\sim 5 \times 5 \mathrm{~cm}^{2} \mathrm{Si}$ wafer substrates. A thin Cr layer was sputtered onto the SLG prior the Si:H layer deposition to ensure adhesion and form a standard reflective interface for further SE measurement. The deposition conditions for the $\mathrm{Si}: \mathrm{H}$ films and the thin Cr layer studied here are summarized in ref. [33] The parallel depositions on $5 \mathrm{~cm} \mathrm{Si}$ wafer substrates were carried out with the same parameters in order to study the layers by cross sectional transmission electron microscopy (XTEM).

The polycrystalline CdS and CdTe thin films were magnetron sputtered onto $\sim 10$ x $15 \mathrm{~cm}^{2}$ Mo-covered soda lime glass (SLG/Mo/CdTe/CdS) [34]

The thin films were measured during deposition in-situ and in real-time using a rotating-compensator multichannel spectroscopic ellipsometer (J.A. Woollam Co., M-2000DI) at one point close to the center of the $15 \times 15 \mathrm{~cm}^{2}$ (Si:H films) or the $10 \times 15 \mathrm{~cm}^{2}$ (CdTe/CdS films) samples. The single-spot measurements of a series of ellipsometric spectra obtained versus time during deposition permit the development of an appropriate multilayer optical model, which provides starting parameters in least-squares regression for the analysis of the mapping data acquired by our prototype spectroscopic ellipsometer for imaging/mapping purposes. To check our mapping expanded beam SE results, we performed mapping SE measurements and evaluations (using the same optical models and wavelength regions) by an AccuMap device (by J.A. Woollam Co) ex-situ.

\section{RESULTS AND DISCUSSION}

The dual-spectral prototype were designed to enable in situ imaging/mapping within one of the chambers of a cluster tool at the Center for Photovoltaics Innovation and Commercialization (PVIC); University of Toledo (Ohio). [31, 32]

\subsection{Calibration and validating measurements}

Calibration and validating measurements were performed on nominally 20, 40, 60 and $80 \mathrm{~nm}$ thermal oxide covered 6inch Si-wafers. The results were compared with AccuMap results, see Figure 2. 


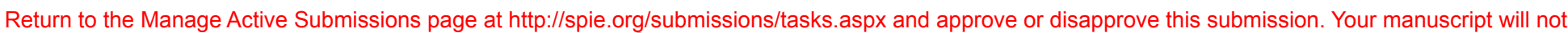
be published without this approval. Please contact author_help@spie.org with any questions or concerns.
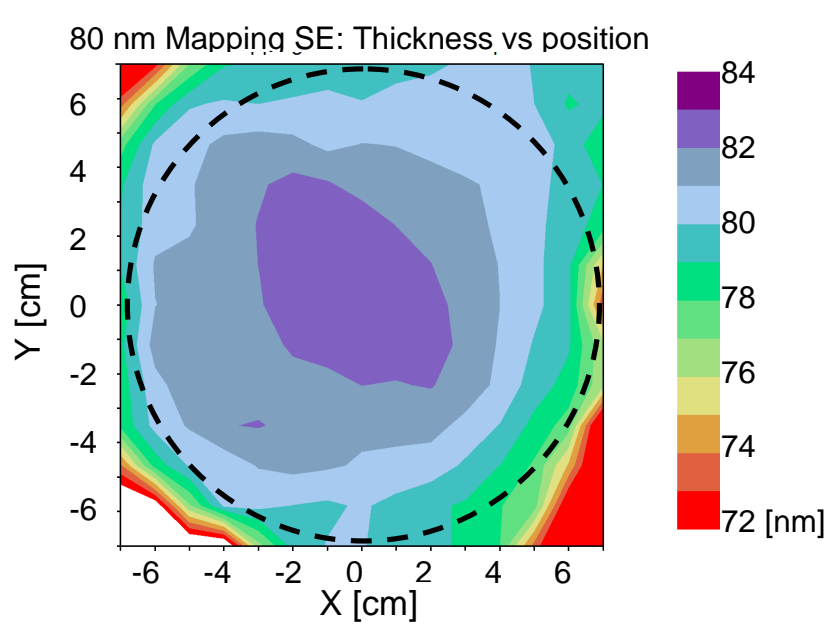

Total Thickness in nm vs. Position

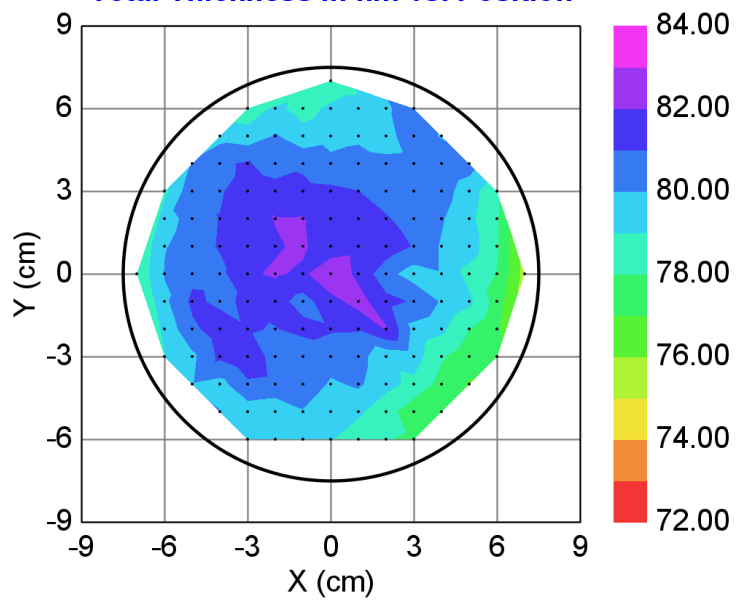

Figure 2 Calibrating and validating measurements on thermal oxides (nominal thickness: $80 \mathrm{~nm}$ ) Note, differences between expanded beam (left) and AccuMap (right) $\leq 1 \mathrm{~nm}$. Only the center line is used for calibration, the other points considered as checking. Points at the corners are from spectra measured on partly the sample and mainly on the holder.

\subsection{Demonstration measurements on $15 \mathrm{~cm}$ sample-size device}

\section{Silicon layers}

Demonstration mapping measurements have been performed ex situ on mixed-phase (a Si:H + nc-Si:H) silicon films having the structure: $\mathrm{nc}-\mathrm{Si} /(\mathrm{nc}-\mathrm{Si}+\mathrm{a}-\mathrm{Si}) / \mathrm{a}-\mathrm{Si} / \mathrm{Cr} /$ glass. Imaging/mapping for initial demonstration purposes, however, was performed on a single phase a $\mathrm{Si}: \mathrm{H} / \mathrm{Cr} /$ glass sample (see Fig. 3) and compared with independent mapping measurements made by a commercially-available instrument (AccuMap) that is capable of point-by-point mapping measurements on larger size samples. With intentionally extreme settings of the deposition parameters, a purely amorphous layer with high spatial non-uniformity was prepared in order to demonstrate the utility of our method. A relatively simple optical model was used to analyze the mapping measurement: opaque $\mathrm{Cr}$ as a substrate, and a-Si:H with a Tauc-Lorentz oscillator dispersion model. The parameters of the Tauc-Lorentz oscillator were taken from the real time SE measurement.
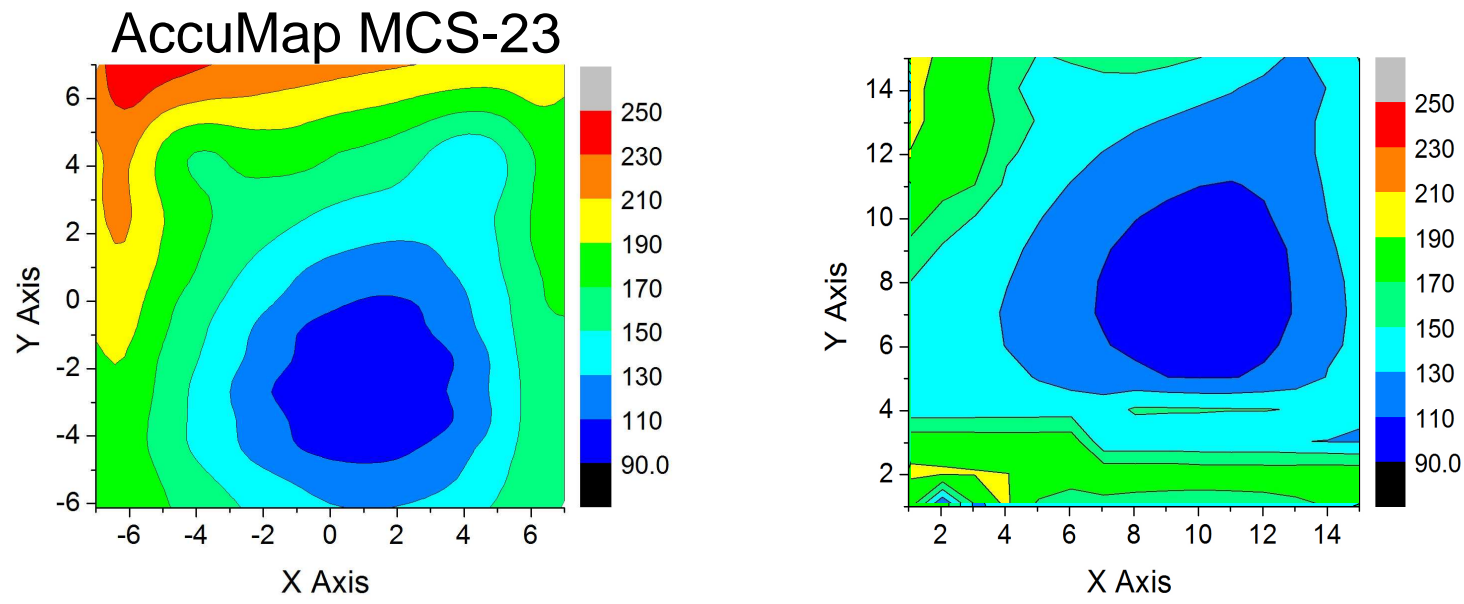

Figure 3 Mapping with a commercial instrument (left) and imaging/mapping with an expanded beam instrument (right) showing reasonable agreement; thickness maps in $\mathrm{nm}$ were obtained for a $15 \mathrm{x} 15 \mathrm{~cm}^{2}$ size sample of single-phase hydrogenated amorphous silicon (a-Si:H) layer on $\mathrm{Cr}$. The map generated by the commercial instrument denotes points on a square grid at the sample surface; the map generated by the expanded-beam ellipsometer is described in terms of pixel-group coordinates. 
Return to the Manage Active Submissions page at http://spie.org/submissions/tasks.aspx and approve or disapprove this submission. Your manuscript will not be published without this approval. Please contact author_help@spie.org with any questions or concerns.
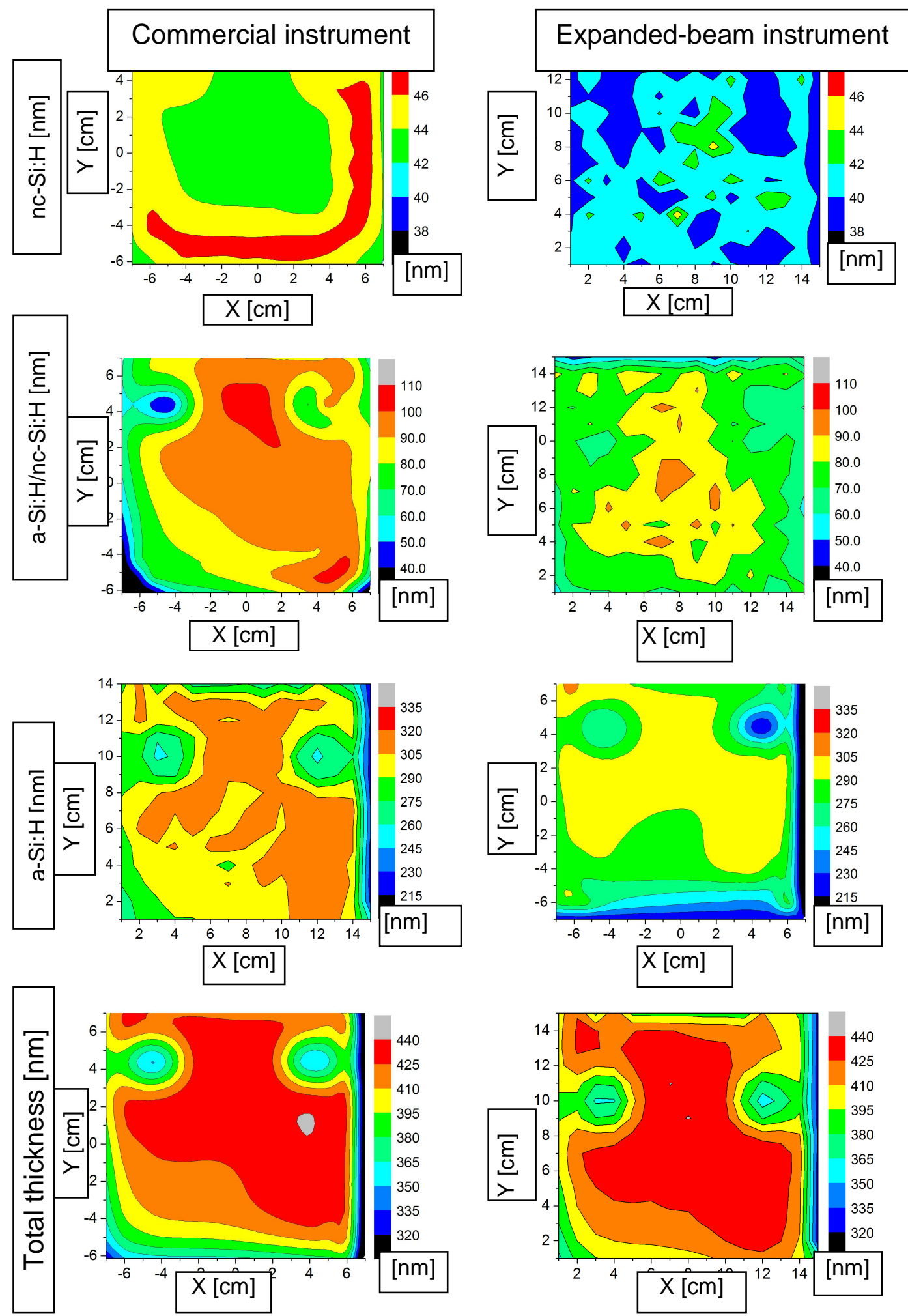

Figure 4 Mapping with a commercial instrument and imaging/mapping with the expanded-beam instrument for an thin Si:H film with $\mathrm{R}=20$ using the 3-layer optical model given by nc Si:H/(a Si:H+nc-Si:H)/a-Si:H /Cr. The total thickness is the sum of the thicknesses of the three sub-layers for each spatial point. 


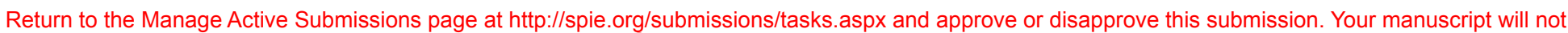
be published without this approval. Please contact author_help@spie.org with any questions or concerns.

We performed the second evaluation of the mapping measurement using the $\mathrm{R}=20\left(\mathrm{H}_{2} / \mathrm{SiH}_{4}\right.$ ratio, see Ref. 33) and applying a 3-layer optical model consisting of the following stack: nc $\mathrm{Si}: \mathrm{H} /(\mathrm{a}-\mathrm{Si}: \mathrm{H}+\mathrm{nc}-\mathrm{Si}: \mathrm{H}) / \mathrm{a}-\mathrm{Si}: \mathrm{H} / \mathrm{Cr}$, which was developed from single-spot, real-time spectroscopic ellipsometry (SE) analysis [33]. In addition to the appropriate model, this real time SE measurement also provided good starting parameters in least-squares regression for the analysis of the mapping data using the model. We also compared our mapping results with the results (using the same optical model) of independently performed ex-situ measurements with the AccuMap (see Fig. 4). We must note that this latter instrument obtains ellipsometric spectra at $\mathrm{mm}$-size spots on a square grid, whereas the expanded-beam instrument obtains spectra for $\sim 5 \times 5 \mathrm{~mm}^{2}$ areas. So, we cannot ensure that the individual ellipsometers are collecting spectra from precisely the same areas with the same resolution.

The agreement in Fig. 4 is very good, considering the complexity of the model, consisting of three individual Si:H layers. The circular areas of reduced thickness on the maps arise from the use of a top cathode plate with two holes that permit passage of the beam for real time SE. When larger area devices are made, these specialized electrodes are replaced with standard ones.

\section{$S L G / \mathrm{Mo} / \mathrm{CdTe} / \mathrm{CdS}$}
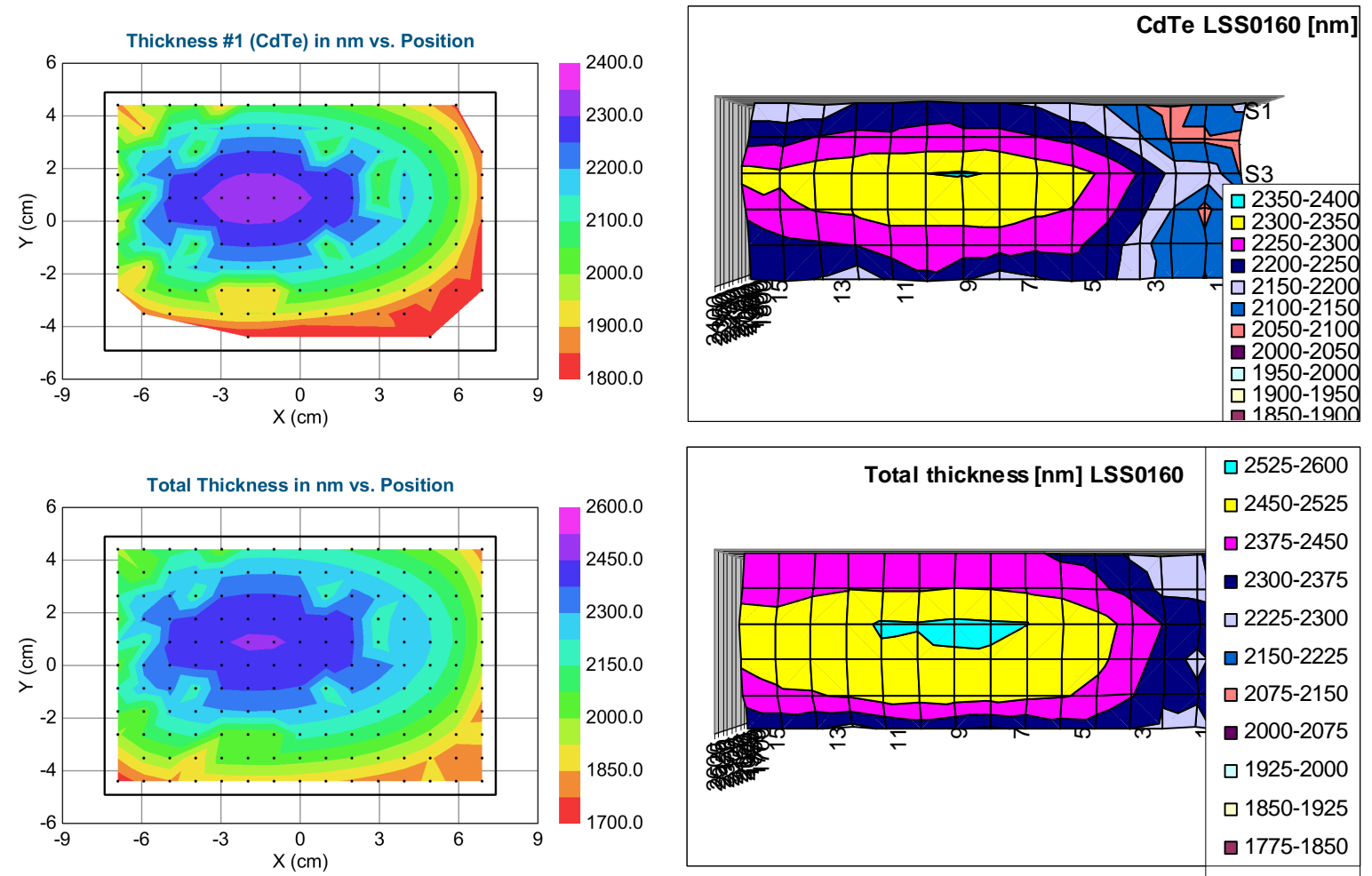

Figure 5 Mapping with the AccuMap (left) and imaging/mapping with the expanded-beam instrument (right) for a SLG/Mo/CdTe/CdS sample. The total thickness is the sum of the thicknesses including the surface roughness and interface layers for each spatial point.

The magnetron sputtered polycrystalline CdS and CdTe thin films were modelled by dielectric functions taken from real time SE measurement and the model contained surface roughness and interface layers, too. We show only the thickness of the CdTe layer and the "Total thickness" (all layers in the models, including the surface roughness and interface layers) in Fig. 5. Unfortunately, we had a problem with the sample holder (because of the continuous changing of the rigid sample holders and the cassette roll-to-roll model machine) so we could measure only the central part of the sample by the imaging instrument.

One remark: if the lateral change (inhomogeneity) is well over $1 \mathrm{~nm} / \mathrm{mm}$ then the expanded beam ellipsometer does not measure the same thing as single spot ellipsometers! In the case of $80 \mathrm{~nm}$ thick $\mathrm{SiO}_{2}$ film the inhomogeneity is: $6 \mathrm{~nm} / 6$ $\mathrm{cm}=0.1 \mathrm{~nm} / \mathrm{mm}$, so the inhomogeneity within the expanded beam SE's elementary area is $0.5 \mathrm{~nm}$. In the case of the polysilicon sample (see Fig. 8) the inhomogeneity is $50 \mathrm{~nm} / 6 \mathrm{~cm}=0.8 \mathrm{~nm} / \mathrm{mm}$, so the inhomogeneity within the 


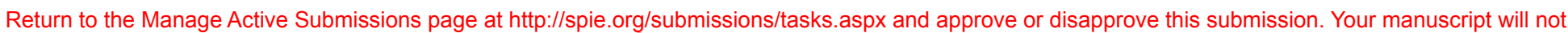
be published without this approval. Please contact author_help@spie.org with any questions or concerns.

expanded beam SE's elementary area is $4 \mathrm{~nm}$. In the case of the CdTe/CdS sample the inhomogeneity is $200 \mathrm{~nm} / 3 \mathrm{~cm}$ $=6.7 \mathrm{~nm} / \mathrm{mm}$, so the inhomogeneity within the expanded beam SE's elementary area is $40 \mathrm{~nm}$ ! However, the difference between the mapping results is below $1 \%$ in all cases.

\section{In-line roll-to-roll (RtR) measurement}

We measured $\mathrm{ZnO}$ layer (deposited by magnetron sputtering onto an Ag-covered plastic foil in a cassette roll-to-roll model machine) and a-Si/ZnO/Ag/plastic-foil structures in-line by the expanded beam device, see ref [32]. We used a simple Cauchy-dispersion for the $\mathrm{ZnO}$ layer because of the limited wavelength region. In the case of the a$\mathrm{Si} / \mathrm{ZnO} / \mathrm{Ag} /$ plastic-foil structure, we measured first the $\mathrm{ZnO}$-layer only (on $\mathrm{Ag}$ layer/plastic foil) RtR sample and moved the cassette roll-to-roll model machine into the a-Si deposition chamber. After the a-Si deposition the cassette roll-to-roll model machine was moved back to the measuring chamber (without breaking the vacuum) and measured again by the expanded beam mapping SE, see Fig. 6. Mapping SE was performed from the leading end of the roll, counter-clockwise (CCW) first and clockwise (CW) after the a-Si deposition. We have got the same map for the ZnO layer and we could see the same greater MSE values at the "wrinkled" line of the foil! This "wrinkle" causes a similar effect as the edges of the foil, possibly making the angles of incidence locally different from the calibrated ones. This effect shows that our expanded beam mapping SE is a robust device which is relatively insensitive for the small distortions. [32]
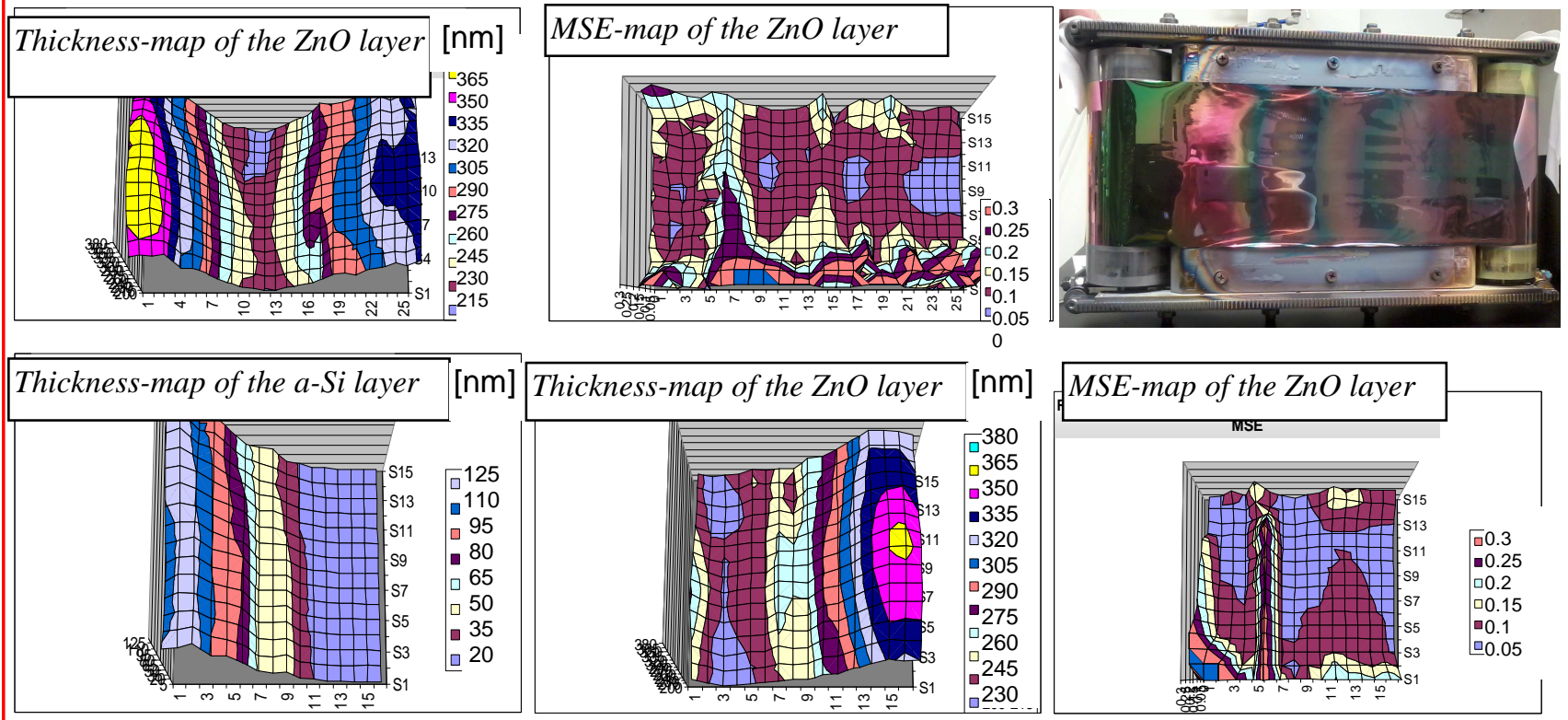

Figure 6 In-line Roll-to Roll: Maps of the $\mathrm{ZnO}$ layer (upper, deposited by magnetron sputtering onto Ag-covered plastic foil in a cassette roll-to-roll model machine, upper-right) and maps (bottom) of a-Si layer, the same ZnO layer and MSE-map (of second measurement). The a-Si layer was deposited after the first measurement. (Axis is in $\mathrm{cm} \mathrm{steps).} \mathrm{Mapping} \mathrm{SE} \mathrm{was} \mathrm{performed} \mathrm{from} \mathrm{the}$ leading end of the roll (CCW and $\mathrm{CW})$. Note, the greater MSE values at the "wrinkled" line of the foil! [32]

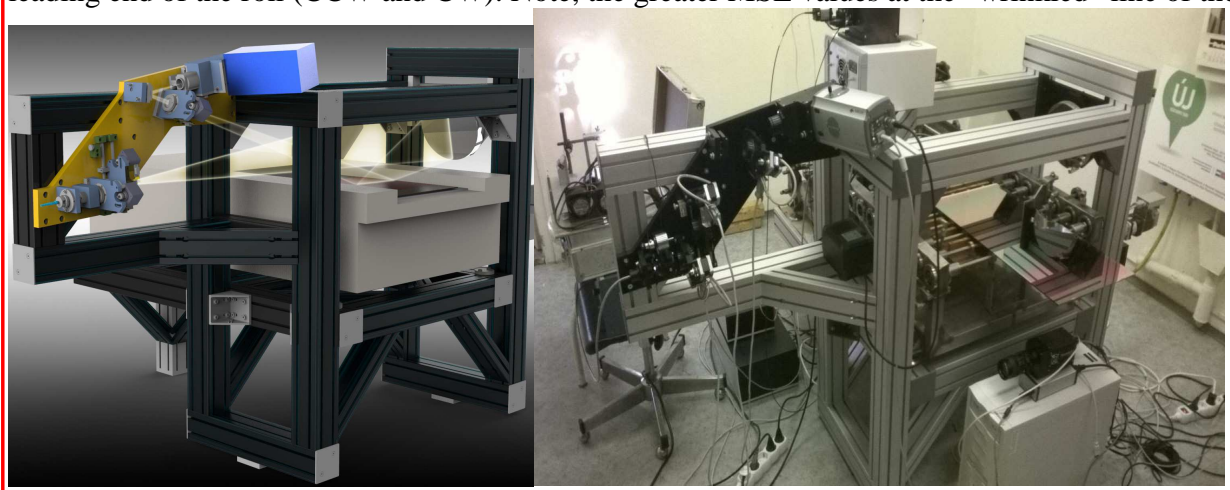

Figure 7 Single-spectrum (350-1000 nm) expanded beam SE mapping device for 30-cm sample-size is completed at MFA (Budapest, Hungary); CAD-drawing with the beam-path (left) and photograph (right) 
Return to the Manage Active Submissions page at http://spie.org/submissions/tasks.aspx and approve or disapprove this submission. Your manuscript will not be published without this approval. Please contact author_help@spie.org with any questions or concerns.

\subsection{Demonstration measurements on $30 \mathrm{~cm}$ sample-size device}

This year, we have built a single-spectrum (350-1000 nm) instrument for 30-cm sample-size at MFA (Budapest, Hungary) and performed demonstration measurements. (See Fig. 7)

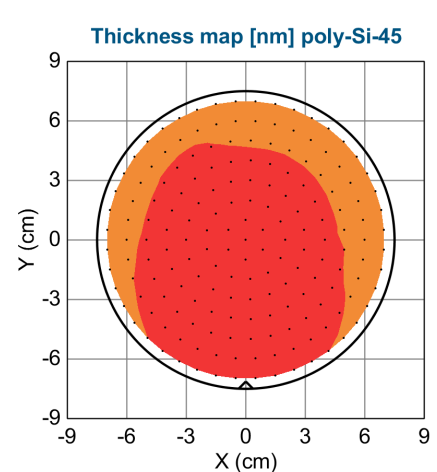

Thicknessmap $[\mathrm{nm}]$ poly-Si-49

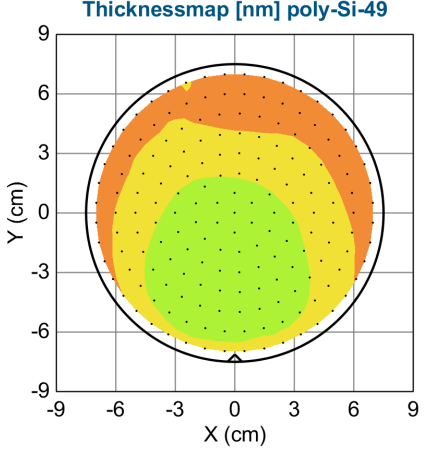

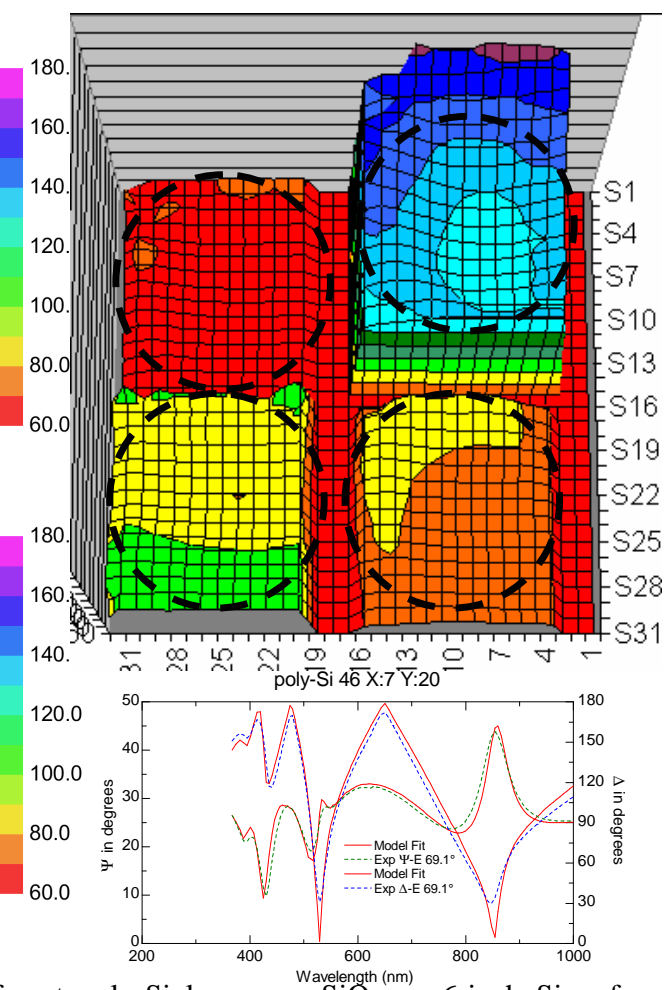
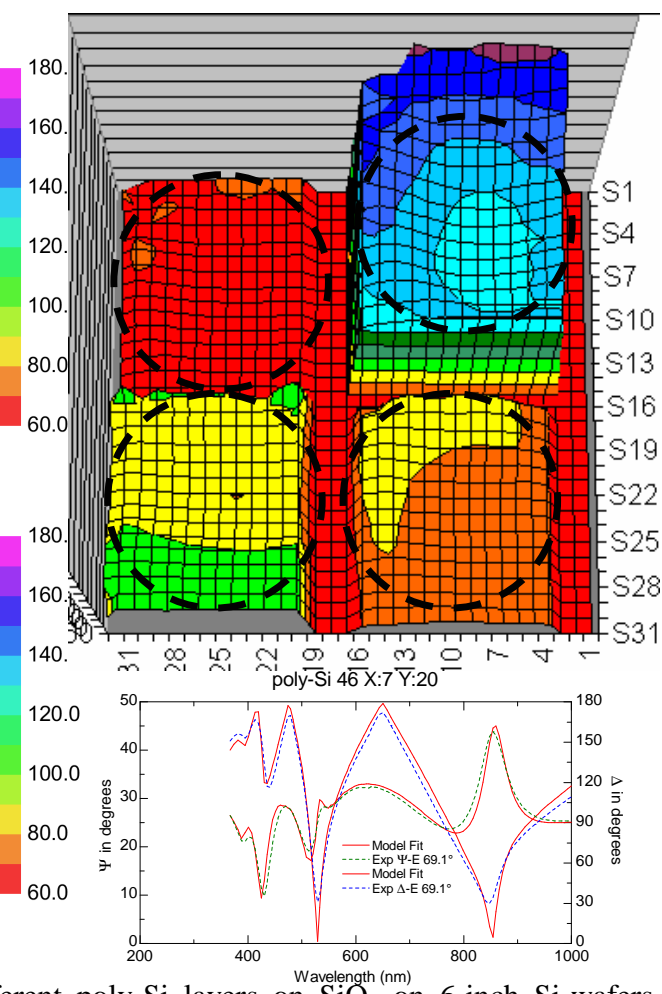

Figure 8 Thickness-map of 4 different poly-Si layers on $\mathrm{SiO}_{2}$ on 6-inch $\mathrm{Si}$-wafers by commercial ellipsometer (left and right, measuring time $\sim 4 \times 20 \mathrm{~min}$.), and by the $30 \mathrm{~cm}$ wide expanded beam device (center, measuring time $\sim 3 \mathrm{~min}$.). $1 \mathrm{color}=5 \mathrm{~nm}$. A sample spectrum-pair (with model fit; optical model: effective medium approximation with c-Si/a-Si mixture) from a central sampleposition is shown (lower center part).

One demonstration is an ,artificial” $30 \times 30 \mathrm{~cm}^{2}$ sample assemblyed from 4 different poly-Si layers on $\mathrm{SiO}_{2}$ on 6-inch Siwafers, see Fig. 8. We checked the thickness-maps by commercial ellipsometer, too. The measurement by the $30 \mathrm{~cm}$ wide expanded beam device are in good agreement with results of the commercial SE device, see Fig. 8.

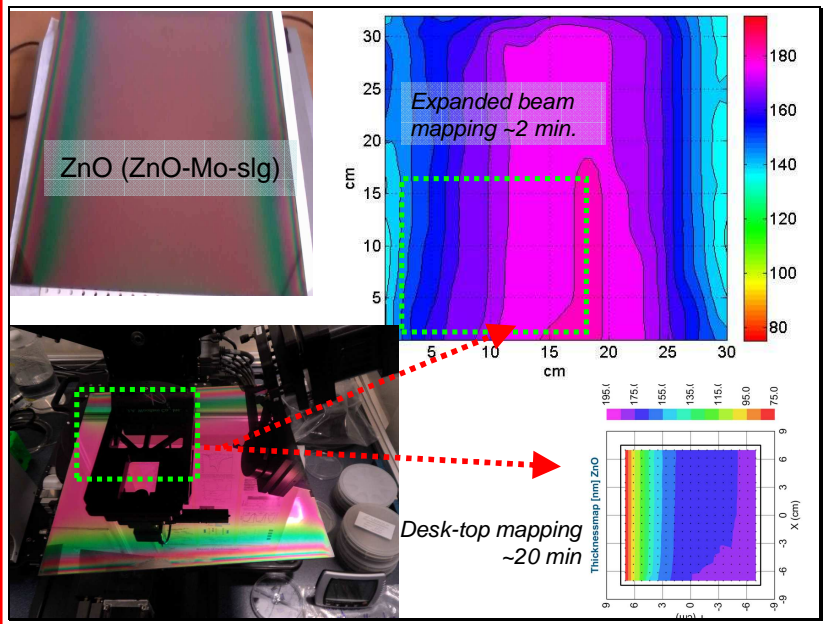

Figure 9 Thickness-map of a $\mathrm{ZnO} / \mathrm{Mo} /$ glass sample by the $30 \mathrm{~cm}$ wide expanded beam device (upper, right, measuring time $\sim 2$ min.). 1 color $=9 \mathrm{~nm}$. We can perform control measurements by a commercial SE only on a $15 \times 15 \mathrm{~cm}^{2}$ part of the sample (dashed rectangular) because of geometrical reason. 


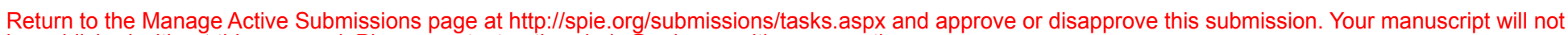
be published without this approval. Please contact author_help@spie.org with any questions or concerns.

Next demonstration is a $\mathrm{ZnO}$ layer on Mo-covered $30 \times 30 \mathrm{~cm}^{2}$ glass. We performed a control (comparison) mappingmeasurement by a commercial SE only on a $15 \times 15 \mathrm{~cm}^{2}$ part of the sample (dashed rectangular) because of geometrical reason. The agreement is very good, see Fig. 9.

We measured more photovoltaic-like samples, too. One is a non-transparent (a-Si/Al/glass), the other is a semitransparent (a-Si/glass, transparent in the red-side of the spectrum) sample. The results on the semi-transparent (a$\mathrm{Si} /$ glass) sample seems to be as reliable as on the non-transparent (a-Si/Al/glass) sample. Thickness-maps by the $30 \mathrm{~cm}$ wide expanded beam device and by the commercial SE device are compared in Fig. 10.

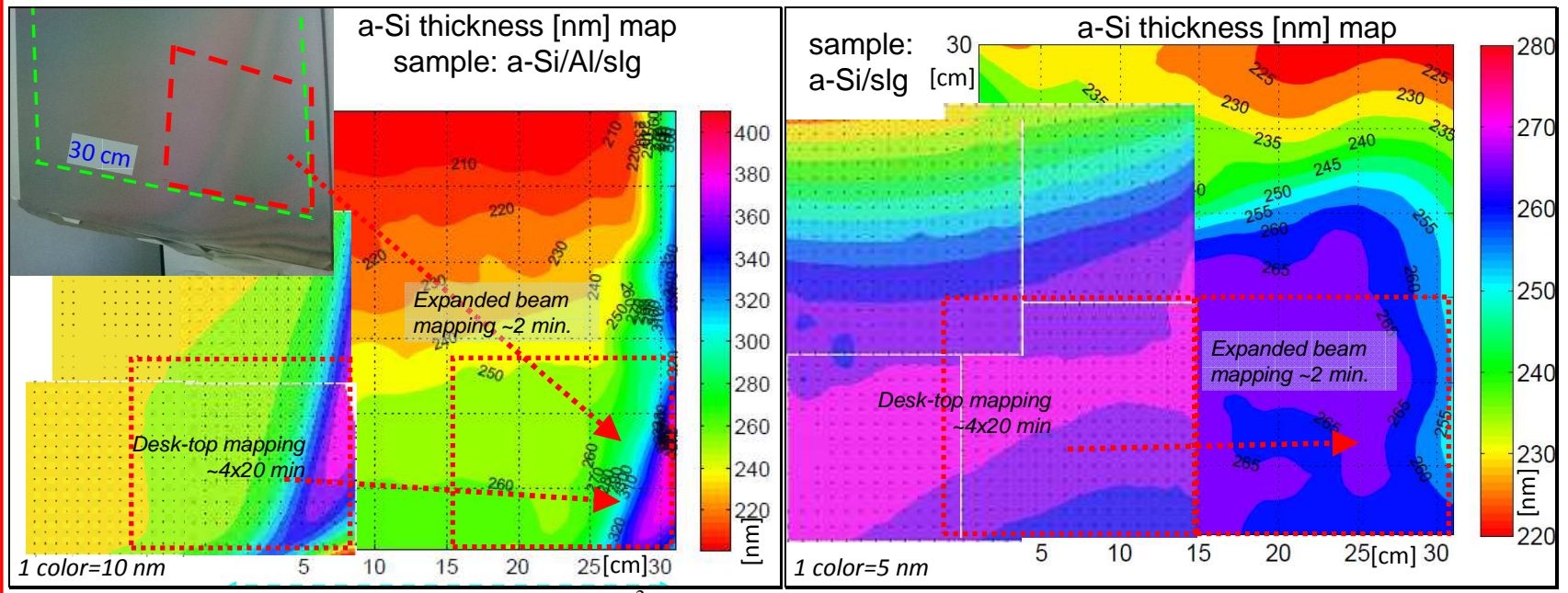

Figure 10 Thickness-maps of a-Si layers on $30 \times 30 \mathrm{~cm}^{2}$ a-Si/Al/glass (left) and a-Si/glass (right) samples by the $30 \mathrm{~cm}$ wide expanded beam device and by a commercial ellipsometer. 1 color $=10 \mathrm{~nm}$. Maps by the commercial ellipsometer are merged from independent $415 \times 15 \mathrm{~cm}^{2}$ maps (dashed rectangulars).

Our group just finished a domestic project (supported by the Hungarian Government) in consortium with a Hungarian company (Tenzi Ltd.). We have developed prototypes for $60-\mathrm{cm}$ and $90-\mathrm{cm}$ sample-size. These instruments can measure the single-spectrum (350-1000 nm) within $5 \mathrm{sec}$.

\section{CONCLUSIONS}

Thin film PV is more cost effective than bulk PV technology, but higher efficiency needs lateral homogeneity! Commercial instruments using 1D detector arrays for spectroscopic ellipsometry must translate either the sample or the ellipsometer in two dimensions in order to map large area samples. A $15 \times 15 \mathrm{~cm} 2$ sample requires $>200$ measurements and at least $15 \mathrm{~min}$ of measurement time to achieve $\mathrm{cm}$ scale spatial resolution. By using a 2D detector array and imaging along one dimension in parallel, the sample need only be translated in one dimension in order to map large area samples. As a result, our expanded-beam system (that provides a line image of spectroscopic (350-1000 $\mathrm{nm}$ ) ellipsometry) data can measure with similar spatial resolution in $<2$ min. (Currently a single 40 point line image can be collected in $10 \mathrm{~s}$ over a $15 \mathrm{~cm}$ width of PV material.) Incorporating the results of real time SE measurements, which provide good starting parameters in least-squares regression for the analysis of the imaging/mapping data, expandedbeam ellipsometry can be an effective in-line monitor of uniformity in PV production lines using roll-to-roll or rigid plate substrates. The goal of near future efforts is to increase the speed by 10x and scale up the width by 2-3x. Then 1800 points could be mapped in a 1 min traverse of a $(60 * 120 \mathrm{~cm}) \mathrm{PV}$ panel.

\section{ACKNOWLEDGMENTS}

This work was supported by the EU ENIAC "E450EDL", EU SEA4KET and National Development Agency KMR_12_1_2012_0225 projects. 
Return to the Manage Active Submissions page at http://spie.org/submissions/tasks.aspx and approve or disapprove this submission. Your manuscript will not be published without this approval. Please contact author_help@spie.org with any questions or concerns.

\section{REFERENCES}

[1] H. Fujiwara, "Spectroscopic Ellipsometry: Principles and Applications", Wiley (2007)

[2] "Handbook of Ellipsometry", H. G. Tompkins, E. A. Irene (eds.), William Andrew Publishing (2005)

[3] „Ellipsometry at the Nanoscale”, M. Losurdo, K. Hingerl (eds.) Springer (2013)

[4] T. Lohner, N. Q. Khánh, Z. Zolnai, Spectroellipsometric characterization of ion implanted semiconductors and porous silicon, Acta Physica Slovaca 48 (1998) 441-450.

[5] M. Serényi, T. Lohner, P. Petrik, C. Frigeri, Comparative analysis of amorphous silicon and silicon nitride multilayer by spectroscopic ellipsometry and transmission electron microscopy, Thin Solid Films 515 (2007) 35593562.

[6] M. Serényi, T. Lohner, P. Petrik, Z Zolnai, ZE Horvath, NQ Khanh, Characterization of sputtered and annealed niobium oxide films using spectroscopic ellipsometry, Rutherford backscattering spectrometry and X-ray diffraction, Thin Solid Films 516(22) (2008) 8096-8100.

[7] P. Petrik, Ellipsometric models for vertically inhomogeneous composite structures, PHYSICA STATUS SOLIDI A 205(4) (2008) 732-738

[8] T. Lohner, A. Szekeres, T. Nikolova, E. Vlaikova, P. Petrik, G. Huhn, K. Havancsak, I. Lisovskyy, S. Zlobin, I. Z. Indutnyy, P. E. Shepeleliavyi, Optical models for ellipsometric characterization of high temperature annealed nanostructured SiO2 films, Journal of Optoelectronics and Advanced Materials 11 (9): (2009) 1288-1291.

[9] A. Szekeres, T. Lohner, P. Petrik, G. Huhn, K. Havancsak, I. Lisovskyy, S. Zlobin, I. Z. Indutnyy, P. E. Shepeliavyi, Ellipsometric characterization of SiOx films with embedded Si nanoparticles, Vacuum 84(1), (2009) 115-118

[10] T Lohner, A Pongracz, NQ Khanh, OH Krafcsik, KV Josepovits, P Deak, Comparative investigation of the Si/SiO2 interface layer containing $\mathrm{SiC}$ crystallites using spectroscopic ellipsometry, ion beam analysis and XPS, PHYSICA STATUS SOLIDI C-CURRENT TOPICS IN SOLID STATE PHYSICS 5(5) (2008) 1337-1340.

[11]P. Kozma, B. Fodor, A. Deak, P. Petrik, "Optical Models for the Characterization of Silica Nanosphere Monolayers Prepared by the Langmuir-Blodgett Method Using Ellipsometry in the Quasistatic Regime", Langmuir 26 (2010) 16122.

[12]P. Petrik, "Characterization of Nanocrystals Using Spectroscopic Ellipsometry", In: Sudheer Neralla (ed.), "Nanocrystals - Synthesis, Characterization and Applications", InTech, 2012, 29-40 (ISBN:978-953-51-0714-9)

[13] L.R. Dahal, Z. Huang, D. Attygalle, C. Salupo, S. Marsillac, N.J. Podraza, R.W. Collins, Correlations between Mapping Spectroscopic Ellipsometry Results and Solar Cell Performance for Evaluations of Non-Uniformity in Thin Film Silicon Photovoltaics, IEEE Journal of Photovoltaics 3(1), art. no. 6336774 , (2013) 387-393

[14]P. Aryal, D. Attygalle, P. Pradhan, N.J. Podraza, S. Marsillac, R.W. Collins, Large-Area Compositional Mapping of $\mathrm{Cu}(\operatorname{In}\{1-\mathrm{x}\} \mathrm{Ga}\{\mathrm{x}\}) \operatorname{Se}\{2\}$ Materials and Devices with Spectroscopic Ellipsometry, IEEE Journal of Photovoltaics 3 (1), art. no. 6311418, (2013) 359-363

[15] A.M. Hermann, C. Gonzalez, P.A. Ramakrishnan, D. Balzar, C.H. Marshall, J.N. Hilfiker, T. Tiwald, Growth and characterization of large area $\mathrm{Cu}(\mathrm{In}, \mathrm{Ga}) \mathrm{Se} 2$ films, Thin Solid Films 387 (2001) 54

[16] M.I. Alonso, M. Garriga, C.A. Durante Rincon, E.Hernandez, M. Leon, Optical functions of chalcopyrite $\mathrm{CuGa}\{\mathrm{x}\} \operatorname{In}\{1-\mathrm{x}\} \mathrm{Se} 2$ alloys, Appl. Phys. A 74 (2002) 659-664

[17] S. Minoura, K. Kodera, T. Maekawa, K. Miyazaki, S. Niki, H. Fujiwara, Dielectric function of Cu(In, Ga)Se2-based polycrystalline materials, Journal of Applied Physics 113 (2013) 063505

[18] “OPTICAL CONTROL OF MULTI-STAGE THIN FILM SOLAR CELL PRODUCTION”, United States Patent Application US/SN 61/778,079, Filed March 12, 2013, Inventors: Jian Li, Dean Levi, Miguel Contreras, John Scharf

[19] J. Li, J. Chen, R. W. Collins, Broadening of optical transitions in polycrystalline CdS and CdTe thin films, APPLIED PHYSICS LETTERS 97 (2010) 181909

[20] J. Li, J. Chen, R. W. Collins, Optical transition energies as a probe of stress in polycrystalline CdTe thin films, APPLIED PHYSICS LETTERS, 99 (2011) 061905

[21]P. I. Rovira, R. W. Collins, Analysis of specular and textured SnO2:F films by high speed four-parameter Stokes vector spectroscopy, Journal of Applied Physics 85 (1999) 2015

[22] H. Fujiwara and M. Kondo, Effects of carrier concentration on the dielectric function of $\mathrm{ZnO}: \mathrm{Ga}$ and In2O3:Sn studied by spectroscopic ellipsometry: Analysis of free-carrier and band-edge absorption, Phys. Rev. B 71 (2005) 075109

[23] M. Akagawa and H. Fujiwara, High-precision characterization of textured a-Si:H/SnO2:F structures by spectroscopic ellipsometry, J. Appl. Phys. 110 (2011) 073518

[24] H. Yoshikawa, S. Adachi, Optical Constants of ZnO, Japanese Journal of Applied Physics 36 (1997) 6237 
Return to the Manage Active Submissions page at http://spie.org/submissions/tasks.aspx and approve or disapprove this submission. Your manuscript will not be published without this approval. Please contact author_help@spie.org with any questions or concerns.

[25]C. Major, A. Nemeth, G. Radnoczi, Zs. Czigany, M. Fried, Z. Labadi, I. Barsony, Optical and electrical characterization of aluminium doped ZnO layers, Applied Surface Science 255 (2009) 8907

[26] S. Kageyama, M. Akagawa, H. Fujiwara; Dielectric function of a-Si:H based on local network structures, Phys. Rev. B 83 (2011) 195205

[27],,IMAGING OPTICAL INSPECTION DEVICE WITH A PINHOLE CAMERA”; Pub. No.: WO/2008/142468; International Application No.: PCT/HU2008/000058 Publication Date: 27.11.2008 International Filing Date: 23.05.2008, US 8437002 B2, Priority Data: P 0700366, 23.05.2007, HU, Inventors: Zoltán György Horváth, György Juhász, Miklós Fried, Csaba Major, Péter Petrik

[28] G. Juhasz, Z. Horvath, C. Major, P. Petrik, O. Polgar, M. Fried, Non-collimated beam ellipsometry, Phys. Stat. Sol. (c) 5 (2008) 1081-1084

[29] C. Major, G. Juhasz, Z. Horvath, O. Polgar, M. Fried, Wide angle beam ellipsometry for extremely large samples, Phys. Stat. Sol. (c), 5 (2008) 1077-1080

[30]C. Major, G. Juhasz, P. Petrik, Z. Horvath, O. Polgar, M. Fried, Application of wide angle beam spectroscopic ellipsometry for quality control in solar cell production, Vacuum 84 (2009) 119-122

[31] M. Fried, G. Juhasz, C. Major, P. Petrik, O. Polgar, Z. Horvath, A. Nutsch, Expanded beam (macro-imaging) ellipsometry, Thin Solid Films 519 (2011) 2730-2736

[32] M. Fried, On-line monitoring of solar cell module production by ellipsometry technique, Original Research Article, Thin Solid Films, 571. (2014) 345-355

[33] R. W. Collins, A. S. Ferlauto, G. M. Ferreira, C. Chen, J. Koh, R. J. Koval, Y. Lee, J. M. Pearce, C. R. Wronski, Evolution of microstructure and phase in amorphous, protocrystalline, and microcrystalline silicon studied by real time spectroscopic ellipsometry, Solar Energy Materials and Solar Cells 78 (2003) 143

[34] J. Li, J. Chen, M. N. Sestak, C. Thornberry, R. W. Collins, Spectroscopic ellipsometry studies of thin film CdTe and CdS: from dielectric functions to solar cell structures, 34TH IEEE PHOTOVOLTAIC SPECIALISTS CONFERENCE, VOLS 1-3 (2009) pp. 1783-1788 